\title{
Aus dem Vorwort zur 22. und 23. Auflage
}

Als der Verlag mit der Bitte an mich herantrat, die Bearbeitung der neuen Auflage des "Lehrbuchs der anorganischen Chemie" von A. F. Holleman zu übernehmen, war ich mir darüber im klaren, daß nur durch eine grundlegende Neuplan ung und Umgestaltung des bei den vorhergehenden Auflagen schon mehrfach ergänzten und verbesserten Stoffes wieder ein modernes Werk von innerer Geschlossenheit und einheitlichem GuB zu schaffen war. Dementsprechend habe ich mich nicht mit einer bloßen Ưberarbeitung des Buches begnügt, sondern den enthaltenen Lehrstoff im Geiste des ursprünglichen „Holleman“ auf der Grundlage heutiger Erkenntnisse völlig neu geschrieben und umgestaltet, so daß ein ganz neues Werk ent. standen ist. Dies konnte insofern verantwortet werden, als das neue Buch nicht die Zahl der übrigen Lehrbücher für anorganische Chemie vermehrt, sondern an die Stelle eines dieser Bücher tritt.

Bei der Niederschrift des Lehrbuches ließ ich mich im einzelnen von folgenden Gedankengängen leiten:

1. Die vielfältigen Probleme der Gegenwart stellen an die Ausbildung des chemischen Nachwuchses höchste Anforderungen. Diese Anforderungen werden nach Beendigung des Krieges mit Sicherheit noch erheblich weiter gesteigert werden müssen. Es wäre daher verhängnisvoll, wenn man von der Seite der Lehrbücher her der zeitgemäß bedingten, vielfach unzureichenden naturwissenschaftlichen Vorbildung des studentischen Nachwuchses durch Herabsetzung des Lehrbuchniveaus ent. gegenkommen wollte. Ganz bewußt wurde dementsprechend davon abgesehen, ein „leichtes“ Buch zu schreiben, und im Gegenteil eine intensive und aufgeschlossene Mitarbeit des Lesers vorausgesetzt. Dies um so mehr, als es sich bei dem vorliegenden Werk zwar um ein Anfänger-Lehrbuch, aber um ein solches für Hochschulen und nicht für Mittelschulen handelt, und als von den Studenten, die sich der Chemie verschrieben haben, eine besondere Veranlagung und Aufgeschlossen. heit für die Probleme der Chemie vorausgesetzt werden kann und muß. Es schadet gar nichts, wenn der Chemiestudierende diese und jene Stelle des Buches zwei oder gar mehrere Male durchdenken oder sich mit diesem und jenem Kapitel etwas „abquälen“ muß. Denn ein Lehrbuch soll ja dem Leser das Denken nicht abnehmen, sondern ihn im Gegenteil dazu anregen, und erfahrungsgemäß wird gerade jenes Wissen meist zum festen Besitz, das in heißem Bemühen errungen wurde. Es ist dabei vielleicht nicht unnötig zu betonen, daß auf eine streng logische, klare und anschauliche Entwicklung aller Begriffe und Tatsachen größter Wert gelegt wurde und chemische Vorkenntnisse nicht vorausgesetzt sind. Der Lehrstoff selbst entspricht im großen und ganzen den Anforderungen, die an der Münchener Universität bereits im anorganisch-chemischen Diplom-Vorexamen gestellt werden.

2. Zur erfolgreichen Ausbildung eines Chemikers an einer Hochschule gehören Vor * lesung, Laboratorium und Lehrbuch. Diese drei Ausbildungsformen bilden eine Drei-einheit und sollen sich gegenseitig nicht ersetzen, sondern ergänzen. Dem- 
entsprechend werden die drei Wege zu dem gemeinsamen Ziel zweckmäßig zwar aufeinander abgestimmt, aber doch voneinander verschieden gestaltet. Ein Lehrbuch darf somit nicht vom Standpunkt einer Vorlesung oder eines Prakti. kums aus beurteilt werden und umgekehrt. Hauptziel eines Lehrbu chs ist die Heraus. arbeitung von Zusammenhängen, die das in Vorlesung und Praktikum Erarbeitete unter gemeinsamem Gesichtspunkt erkennen und verstehen lassen. Deshalb wurde im vorliegenden Buch Wert darauf gelegt, Zusammengehörendes auch im Zusammenhang darzubringen. So werden beispielsweise die zur Aufstellung des A tom- und Molekülbegriffs führenden Gesetze und Erkenntnisse nicht wie in den meisten Lehrbüchern der anorganischen Chemie in den Gesamtstoff eingestreut und so im Gedankengang zerrissen, sondern in geschlossener Darstellung (S. 3-30) behandelt. Ebenso werden z. B. alle mit dem Problem des chemischen Gleich. gewichts (S. 100-120), der Elektronentheorie der Valenz (S. 13ð-162) oder der Oxydation und Reduktion (S. 163-176) zusammenhängenden Fragen geschlossen dargestellt, auch auf die Gefahr hin, daß der Anfänger beim erstmaligen Durcharbeiten notgedrungen manches als noch schwerverständlich überschlagen muß. Das Lehrbuch bietet ja zum Unterschied von der freien Vorlesung jederzeit die Möglich. keit des Vor - und Rückblätterns, so daß Stellen, die beim ersten Male nicht ganz „,verdaut" wurden, später — nach Vertiefung der Kenntnisse - mit größerem Erfolg nochmals erarbeitet werden können. Die hier gewählte geschlossene Darstellung der Hauptfragen zwingt dabei den Benutzer, das gerade in Frage stehende Problem wieder im Zusammenhang des übergeordneten Problems und nicht als los. gelöstes Einzelproblem zu betrachten.

3. Die Valenzstrichformeln haben sich in der anorganischen Chemie als weit. gehend unzulänglich, ja vielfach geradezu als falsch und irreführend erwiesen. Trotzdem bedienen sich weitaus die meisten anorganischen Lehrbücher nach wie vor dieses Hilfsmittels. Demgegenüber sind neuere Lehrbücher der anorganischen Chemie in das andere Extrem verfallen, die Valenzstrichformeln völlig auszuschalten, ohne an ihre Stelle etwas Gleichwertiges oder Besseres zu setzen. Das vorliegende Lehrbuch ist erstmals völlig auf der Grundlage der modernen Elektronentheorie der Valenz aufgebaut, deren Folgerungen bezüglich der chemischen Bindung und der Elektronenformeln schon verhältnismäßig fr üh in einem Sonderkapitel (S. 145 -162) in einer für den Anfänger geeigneten Weise entwickelt werden. Auf diese Weise wird der Student frühzeitig in die Denkweise der Elektronentheorie eingeführt und vor Denkfehlern (z. B. bezüglich der Doppelbindung) bewahrt, die erfahrungsgemäß später nur schwer und mühevoll wieder auszurotten sind.

Auch in der Frage des Periodensystems der Elemente weicht das vorliegende Buch etwas vom Herkömmlichen ab. Zweifellos ermöglicht das Periodensystem eine didaktisch klare und einprägsame Anordnung des anorganischen Wissensstoffes. Es sollte daher an möglichst früher Stelle eines Anfängerlehrbuchs entwickelt werden. Dem steht aber die etwas schwierige Ableitung der gebräuchlichen Kurz- und Langperioden-Form des Systems entgegen, so daß das Periodensystem in den meisten anorganischen Lehrbüchern erst an verhältnismäßig später Stelle erscheint. Im 
vorliegenden Lehrbuch wird erstmals von dem — viel zu wenig bekannten und angewandten - gekürzten Periodensystem der Elemente Gebrauch gemacht, das infolge seiner Klarheit und Übersichtlichkeit bereits sehr früh (S. 66-69) abgeleitet werden kann, den Zusammenhang mit dem Atombau für den AnfängeI viel leichter und einleuchtender darstellen läßt (S. 135-140) und später zwanglos zu den bekannten Formen (S. 439-449) bzw. einer neuartigen, leistungsfähigen Form (s. Schlußtafel) des Gesamtperiodensystems der Elemente ergänzt werden kann.

4. Die Kenntnis der Grundlagen und die Möglichkeit der Anwendung physikalisch-chemischer Hilfsmethoden gehören heute zu dem unerläßlichen Rüst. zeug eines modernen Anorganikers. Daher sind Methoden wie der RamanEffekt (S. 313-31S), die Magnetochemie (S. 491-500) usw. im vorliegenden Lehrbuch gebührend berücksichtigt worden. Stets wurde dabei das betreffende Problem nicht vom Standpunkt des Physikochemikers oder Physikers, sondern vom Standpunkt des Anorganikers aus betrachtet, der sich vornehmlich dafür interessiert, was diese Methoden zu leisten vermögen. Auch sonst wurde Wert darauf gelegt, in zusammenfassenden Darstellungen den Leser, soweit dies in einem Anfängerlehrbuch möglich ist, mit den modernen Problemen der anorganischen Chemie - wie z. B. dem aktiven Zustand der festen Materie (S. 391-397), der Silicatstruktur (S. 325-330), dem Atombau (S. $135-145$ S. 555-565), der natürlichen und künstlichen Elementumwandlung (S. 566-608) usw. - vertraut zu machen.

Daneben wurden die technischen Verfahren der chemischen Industrie nirgends vernachlässigt, sondern in aller A usführlichkeit — vgl. z. B. die Schwefel. säuredarstellung (S. 205-208), die Ammoniaksynthese (S. 224 - 227), die Aluminium. erzeugung (S. $379-383$ ), die Natronlaugegewinnung (S. 423-425), den Hochofenprozeß (S. 527-533) usw. - behandelt, um dem Leser den Blick auch für diese Fragen zu öffnen und ihn zu weiterem Buchstudium anzuregen.

5. Eine gute Abbildung besagt oft mehr als eine ganze Seite Text. Daher wurde besonderer Wert auf eine reiche A usstattung des vorliegen den Buches mit didaktisch klarem und einprägsamem Bildmaterial gelegt. So sind nahezu alle 154 Abbildungen neu entworfen und gezeichnet worden. Dem gleichen Ziel der größeren didaktischen Übersichtlichkeit dient die drucktechnische Anordnung des Lehrstoffs, indem durch vielseitige Anwendung von Fett-, Sperr-, Schräg- und Kleindruck das Wesentlich e gegenüber dem weniger Wesentlichen hervorgehoben und Blickpunkte für eine leichtere Orientierung innerhalb des Buches geschaffen wurden. Ebenso soll die bei verschiedenen Verbindungsklassen angewandte neuartige Systematik (vgl. z.B. S. 202203, S.263-265) zur leichteren gedächtnismäßigen Einprägung des Lehrstoffs beitragen.

So ist, hoffe ich, ein Anfängerlehrbuch entstanden, das in vielen Einzelheiten vom Herkömmlichen abweicht und das auf verhältnismäßig begrenztem Raum einen umfangreichen Wissensstoff in weitgehend vollständiger, moderner und didaktisch abgewogener Darstellung vermittelt.

Herzlichen Dank schulde ich meiner lieben Frau für ihre wertvolle Mitarbeit bei der Anfertigung des umfangreichen Registers.

München, im November 1942.

Egon Wiberg 